\title{
Campo organizacional y cambio institucional en el Hospital Psiquiátrico del Valle entre 1956 y 1970 '
}

\author{
Organizational and Institutional change in the \\ Psychiatric Hospital of the Valle 1956-1970
}

\author{
Jose Fernando Sanchez Salcedo \\ Universidad del Valle, Colombia \\ (Rec: marzo de 2019 - Acept: enero de 2020)
}

\begin{abstract}
Resumen
La construcción de un Hospital Psiquiátrico moderno en el Departamento del Valle entre 1956 y 1961 supuso importantes transformaciones en el campo organizacional y en el sistema de creencias sobre la salud mental en la región del sur occidente colombiano. A nivel organizacional, la Junta Directiva del Asilo logró aglutinar esfuerzos de instituciones privadas y públicas en pro del mejoramiento de la atención de los enfermos mentales. Así mismo, con el apoyo del Departamento de Psiquiatría de la Universidad del Valle, cambió la praxis médica sobre la enfermedad mental e hizo del nuevo hospital, un espacio para la formación de especialistas en psiquiatría. En lo que respecta al sistema de creencias, las nuevas políticas implementadas por la OMS sobre la salud mental y el descubrimiento de nuevos tratamientos, operó cambios profundos en los modos de entender la enfermedad. Siguiendo una línea de investigación historiográfica que utilizó como fuente el archivo administrativo del Hospital, el presente trabajo describe el proceso de cambio institucional que se llevó a cabo en el Asilo, en su proceso de transición de Asilo a Hospital Psiquiátrico en Cali entre 1956 y 1960.

Palabras claves: Cambio institucional, campo organizacional, sistema de creencias, hospital, psiquiatría.
\end{abstract}

\begin{abstract}
The construction of a modern Psychiatric Hospital in the Department of Valle led to significant transformations in the organizational field and the belief system about mental health in the region. At the organizational level, the Board of Directors of the Asylum managed to unite efforts of private and public institutions to improve the care of the mentally ill. Likewise, with the support of the Department of Psychiatry of the University of Valle, the medical practice on mental illness changed, and the new Hospital turned into a space for the training of specialists in psychiatry. Regarding the belief system, new policies implemented by WHO on the mental health and the discovery of new treatments for dementia brought about profound changes in the ways of understanding the disease. Following a line of historiographical research that used the Hospital's administrative file as a source, this work describes the process of institutional change that took place in the Asylum in its transition process from Asylum to Psychiatric Hospital in Cali between 1956 and 1960.
\end{abstract}

Keywords: Institutional change, organizational field, system of beliefs, hospital, psychiatry. 


\section{Introducción}

Entre 1950 y 1960 se llevó a cabo en la ciudad de Cali la construcción de un Hospital psiquiátrico moderno que sustituyó al Asilo San Isidro creado en 1937 como correccional y habilitado, luego en 1940, como "manicomio". Este cambio institucional se dio en un periodo de importantes transformaciones que modificaron las concepciones sobre la locura, su diagnóstico y tratamiento. Entre los aspectos más importantes que caracterizaron este periodo se destacan: la nueva concepción sobre la enfermedad mental que acuña la OMS en $1949^{2}$, que enfatizó el carácter preventivo y la necesidad de integrar la práctica psiquiátrica a las demás ramas de la medicina; el lanzamiento de un manual de diagnósticos psiquiátricos DSM I (APA, 1952) y DSM II (APA, 1968) que propone una nueva clasificación de enfermedades estrechamente ligadas a la corriente biomédica que va a caracterizar a la psiquiatría norteamericana y que termina imponiéndose a la vieja tradición clínica alemana y francesa. En este contexto, el principal aporte que definirá la supremacía norteamericana será la revolución farmacológica que generará, paradójicamente, un descubrimiento francés, la clorpromazina, para el tratamiento de la esquizofrenia y de otras enfermedades mentales.

Todos estos aspectos influenciaron la práctica médica del Departamento de Psiquiatría a partir de 1955, adscrito a la recién creada Facultad de Medicina de la Universidad del Valle en 1950, pues al igual que otras instituciones y facultades de medicina del país, esta contó con el apoyo de la Fundación Rockefeller, quien garantizó tanto apoyo económico, como un importante direccionamiento académico para la formación de médicos psiquiatras en universidades norteamericanas. El Departamento de psiquiatría cumplió un papel preponderante en el diseño arquitectónico y posterior organización del hospital, pero su principal influencia estaría en la institucionalización de un conjunto de saberes y prácticas médicas que cambiarían paulatinamente el modo de tratar la enfermedad mental.

El presente estudio busca dar cuenta de dichos cambios a través del análisis de los archivos administrativos, lo que supuso la revisión y sistematización de diferentes tipos de documentos administrativos, como actas, resoluciones, correspondencia, informes, etc. Tales materiales fueron fotografiados y categorizados con base a temas. Una vez llevado a cabo el proceso de categorización, la información obtenida se organizó en una base de datos en el programa Acces. Como el propósito del estudio fue indagar los cambios en las prácticas y sistemas de creencias, buena parte de las categorías se centraron en dichos temas, así como en aspectos relacionados con las actividades desarrolladas por la Junta proconstrucción del hospital, el diseño y la construcción del Hospital, el modo en que cambió su estructura organizativa, el personal contratado y la estrategia de atención.

El artículo está dividido en tres partes: la primera presenta los referentes teóricos utilizados en el estudio; la segunda, da cuenta del proceso de transformación de Asilo a Hospital psiquiátrico entendido como un cambio institucional en el que se resalta la conformación de un campo organizacional que lo posibilita. En ese mismo apartado, se plantea un análisis del papel desempeñado por la prensa en la inserción del problema de la locura en la agenda pública local, así como las transformaciones que a nivel administrativo demanda el nuevo hospital y finalmente se proponen algunas conclusiones.

\section{La constitución del Hospital Psiquiátrico San Isidro}

\section{La dinámica del cambio institucional.}

En las últimas décadas del siglo XX ha habido un renovado interés por el estudio de las instituciones en Ciencias Sociales ${ }^{3}$ (March y Olsen, 1984, 1997; Torres, 2015). Esta tendencia que se ha reunido bajo el rótulo de neoinstitucionalismo ayuda a estudiar las funciones organizacionales en contextos (March y Olsen, 1984) y a "establecer relaciones entre los factores culturales y sociales y las diversas formas de organización humana [...] que nos permite comprender los procesos de gestación y adopción de decisiones políticas, en su evolución y secuencia histórica" (Farfán, 2007, p.88).

Aunque son diversas las perspectivas que abarca el neoinstitucionalismo: económico, político, histórico o sociológico, sus autores coinciden en definir las instituciones "como las reglas de juego en una sociedad o, más formalmente, los constreñimientos u obligaciones creados por los seres humanos que le dan forma a la interacción humana" (North, 1990, p. 3). Estos patrones sociales tienden a reproducirse, porque tienen que ver con la manera en que los individuos hacen las cosas en su vida cotidiana, en la cual los seres humanos "actúan como si siguieran reglas; saben cómo hacer las cosas y ese saber incluye una experiencia práctica, una experiencia reflexiva y una orientación normativa" (Escalante, 1992, p. 30).

El origen de estos patrones de comportamiento es de gran importancia histórica porque contribuye a estructurar la política, tanto a nivel de las estrategias como de los objetivos mismos y las posibilidades de consenso y conflicto.

El nuevo institucionalismo aborda lo histórico como un proceso de cambio institucional continuo, en el que desde la división más elemental del trabajo, hasta la constitución de los Estados modernos, se han establecido cuerpos cada vez más complejos de rutinas de comportamiento o reglas de juego que surgen para reducir la incertidumbre existente en la interacción entre los entes sociales [...] la repetición durante periodos prolongados en el tiempo de estas rutinas constituye el mundo de las instituciones (Powell y DiMaggio, 1999, p. 18).

Lo anterior, pese a que como lo afirman posteriormente March y Olsen (2006) "las instituciones cambian con el tiempo en respuesta a la experiencia histórica, pero los cambios no son inmediatos y tampoco garantizan mejoramiento en su capacidad de adaptación y supervivencia, ni su eficiencia" (p. 11). Para comprender el cambio institucional, es preciso identificar el tipo de actores que existen en la sociedad y la relación que estos establecen con su entorno institucional.

En este estudio se abordará el campo organizacional que define la posibilidad de un cambio institucional y el papel que

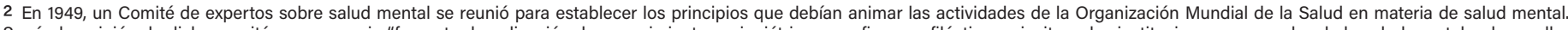

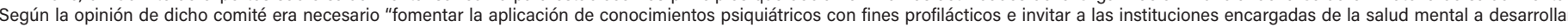

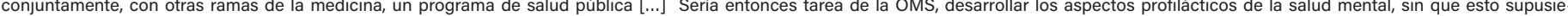

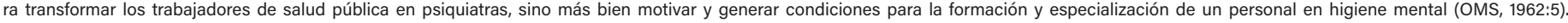


juegan en dicho proceso de cambio las transformaciones en los sistemas de creencias. El campo organizacional se estructura en 4 etapas:

1) aumento de grado de interacción entre las organizaciones en el campo; 2) El surgimiento de estructuras interorganizacionales de dominio y de patrones de coalición claramente definidos; 3) Un incremento de la carga de información de la que deben ocuparse las organizaciones que participan en el campo; y 4) el desarrollo de la conciencia entre los participantes de un conjunto de organizaciones que están en una empresa común (Muñoz, 2014, p. 413).

La importancia del concepto de campo radica en que busca privilegiar los vínculos entre las organizaciones, los cuales sólo pueden establecerse a nivel empírico. Los campos organizacionales se encuentran enmarcados en sistemas de creencias, cuyas ideas influyen "en la forma como los actores perciben sus intereses y opciones en primera instancia, incluyendo las instituciones que preferirían continuar y mantener bajo circunstancias diversas" (Campbell, citado por Muñoz, 2014, p. 409). De esta manera, "los actores dentro de un campo organizacional crean y reproducen las lógicas de un campo" (Yepes, 2017, p. 22). Estas lógicas expresadas en formas comunes de ver y entender problemas y modos de solución van a estar soportadas en reglas, normas y hábitos de comportamiento idénticos o parecidos a otras organizaciones (López, 2009), cuya homogeneidad contribuirá a la difusión unificada de las nuevas creencias que los actores del campo quieren establecer.

La adscripción en un sistema de creencias, permite aglutinar a las organizaciones a partir de puntos de vista e intereses comunes, generando de esta forma procesos de homogenización. Este proceso -en el que las organizaciones buscan parecerse a otras cuando comparten las mismas condiciones ambientales-, ha sido definido como isomorfismo por Powell y Dimaggio (1999). El isomorfismo, entendido como "un proceso limitador que obliga a una unidad en una población a parecerse a otras unidades que enfrentan las mismas condiciones ambientales (Powell y Dimaggio, 1999, p. 108) se puede manifestar de dos formas a nivel competitivo o institucional. El competitivo asociado a "la competencia en los mercados, el cambio de nichos y las medidas de ajuste, y el institucional [...], al poder político y la legitimidad institucional tanto para los ajustes económicos como para los institucionales" (Yepes, 2017, p. 22).

El isomorfismo institucional supone mecanismos de presión de carácter coercitivo, mimético y normativo, que generan el cambio institucional: "1) el isomorfismo coercitivo, que se debe a influencias políticas y al problema de legitimidad; 2) el isomorfismo mimético, que resulta de respuestas estándares a la incertidumbre, y 3) el isomorfismo normativo, asociado con la profesionalización" (Powell y Dimaggio, 1999, p. 109).

Las actividades realizadas por las organizaciones y personas que conforman el campo organizacional generan cambios en dimensiones específicas de la organización como: la infraes- tructura, la estructura formal de la organización, la tecnología, los procesos, los servicios, la cultura organizacional y el comportamiento humano.

En este estudio la transformación del Asilo San Isidro en un Hospital Psiquiátrico se entiende como un cambio institucional producto de las constantes denuncias de la prensa e instituciones del Estado, como la Beneficencia Pública y la Dirección de Higiene del Valle del Cauca, sobre la falta de organizaciones adecuadas para la atención de "los locos" en la ciudad y principalmente en lo relacionado con los costos que debía pagar el gobierno a nivel local por el traslado de dichos enfermos a los Hospitales de Sibaté en Bogotá y San Rafael en Pasto. La identificación de este problema, permitió generar una oportunidad de cambio que se tradujo en la conformación de un campo organizacional (Junta pro construcción del Hospital), compuesto por una serie de actores, que representaban instituciones públicas y privadas de la ciudad.

La acción de la Junta que inicialmente se centró en mejorar las condiciones del Asilo, buscó la financiación para la construcción del nuevo Hospital mediante la realización de una serie de actividades de consecución de recursos, la mayoría de ellas centradas en acciones benéficas, pero sobre todo, difundió -con el respaldo de la prensa local-, una nueva forma de entender el problema de la salud mental en la ciudad que fue generando cambios paulatinos en los procesos de atención y tratamiento de los enfermos en el Asilo hasta el desarrollo de un Hospital con una nueva infraestructura física y tecnológica, nuevos procesos de atención y servicio y funciones diferenciadas del personal médico y administrativo.

Como lo muestra María del Pilar Peralta (2017) en su investigación, periódicos como "Relator", "Diario del Pacífico" introdujeron en su agenda durante la década de los cincuenta, noticias relacionadas con la problemática de los "locos", "dementes" o "enfermos mentales" ${ }^{4}$. Estas noticias contribuyeron a generar un espacio de discusión sobre la problemática en la ciudad, que fue aprovechada por la Junta para sensibilizar a los vecinos de Cali sobre la necesidad de transformar la práctica médica que hasta el momento se llevaba a cabo en el Asilo San Isidro.

La difusión de información sobre el problema de la salud mental contribuyó a la vez, a fortalecer la labor de la Junta y legitimar su acción entre las autoridades locales, al punto que consiguió involucrar a un alcalde entre sus miembros, pero sobre todo intentó generar una nueva forma de abordar el problema de la enfermedad mental, que influyó en los propios miembros de la Junta y en sus redes de relaciones, facilitando de esta forma el cambio a nivel institucional.

De los aspectos mencionados, el éxito más notable de la Junta es que supo involucrar a la recién creada Facultad de Salud de la Universidad del Valle en la asesoría científica de la construcción del Hospital y posteriormente integró a su personal médico en la dirección administrativa y atención clínica de los pacientes. 
El papel de los actores del campo organizacional generó un proceso de isomorfismo institucional, pues creó las condiciones para que el nuevo Hospital adoptara fórmulas institucionales aceptadas y reconocidas, sobre todo en el ámbito de las instituciones de salud mental norteamericanas. En este aspecto, como ya se mencionó, jugaron un importante papel psiquiatras como el Dr. Carlos León, primer jefe del recién creado Departamento de Psiquiatría, que se formó en Tulane y cuyas ideas sobre la atención hospitalaria forjadas en los Estados Unidos, influenciaron el diseño y posterior construcción del Hospital Psiquiátrico.

\section{Del Asilo al Hospital.}

A diferencia de otras ciudades del país, donde existían instituciones asilares para el tratamiento de los enfermos mentales, en Cali, eran recluidos en la Casona San Isidro ${ }^{5}$, la cual fungió como correccional de menores, hasta que en 1940 la Asamblea Departamental del Valle mediante la Ordenanza No 26 de junio de ese mismo año, designó un presupuesto de $\$ 36.000$. oo para la "construcción de un manicomio para la atención y mejoramiento de dementes del Departamento". En un texto dedicado al doctor Carlos León, los psiquiatras José Ramón Jaramillo, Juan Manuel Valverde y Gloria de Jaramillo relatan sus propias impresiones del Asilo hacia 1950:

El Asilo San Isidro era la misma casona de la correccional Meléndez con sus enormes salas de gruesas y altas paredes en las que se disponían hileras de camas de hierro más o menos destartaladas por los "locos mansos" o "dementes inofensivos", como los llamaban los inspectores de policía o los curas párrocos en las cartas de remisión que enviaban con los familiares de los enfermos. Para los "locos furiosos" o simplemente poco colaboradores, había varias hileras de celdas de ladrillo, de más o menos $1.80 \mathrm{~cm}$ de largo por $2 \mathrm{~m}$ de altura y $1 \mathrm{~m}$ de ancho, con una angosta cama de cemento desnuda que ocupaba casi todo el espacio y una puerta de reja de gruesos barrotes que se cerraba por fuera con un candado. La comida se les pasaba por entre las rejas y en la celda hacían sus necesidades fisiológicas como animales enjaulados. En el exterior de las celdas podían verse argollas empotradas en el piso, a las que estaban fijas las cadenas y los grillos con los que se aseguraban a "los locos bravos" cuando no estaban encerrados en las celdas. En el resto del edificio había amplios patios, hermosos jardines, un huerto bien cuidado y un chiquero con varios cerdos obesos y saludables" (Jaramillo, SF: 1).

Antes de la existencia de San Isidro, los enfermos mentales eran al igual que los lisiados e incapacitados para trabajar, albergados en la cárcel pública (Hurtado, 1996). En 1910, según una crónica del periódico El Relator se inauguró un "manicomio-cárcel", ubicado en la Avenida Uribe Uribe entre las calles 20 y 21. Sin embargo, después de muchas discusiones entre las que se destaca "que las condiciones climatéricas (sic) de la ciudad no eran propicias para el establecimiento de un hospital de esa naturaleza [...] se resolvió convertirlo en cárcel. Un edificio construido a la loca, sin método alguno, como que para locos era construido" 6 .
Los enfermos que corrían con mejor suerte eran enviados a Sibaté o a San Rafael en Pasto, los demás eran encerrados inicialmente en la cárcel y después en la correccional de menores que se creó entre 1937 y 1938, hasta que en 1944 se conformó el Asilo Meléndez. Esta nueva institución fue asignada a las Hermanas de la Caridad de San Vicente de Paul, las cuales eran las encargadas de la asistencia y sostenimiento de los enfermos y en general de su funcionamiento. En un contrato firmado entre las Hijas de la Caridad de San Vicente de Paul y la Alcaldía Municipal de Cali, en 1946 se les autoriza la total dirección del servicio interno del Asilo Meléndez ${ }^{7}$. Entre las funciones asignadas están: recibir el dinero enviado por el municipio; hacer las compras y los gastos que requiera la asistencia de los enfermos y empleados; llevar los libros de cuentas; registrar las defunciones de los enfermos; suministrar la medicina y alimentación ordenada por los médicos; admitir o despedir los empleados a su cargo; velar por la moralidad, orden, aseo y policía del establecimiento; tener bajo su autoridad a los enfermos y empleados.

Las hermanas como lo relata Jaramillo (1970), "eran ayudadas por empleados de ambos sexos, algunos de ellos expacientes o pacientes inofensivos sin ningún conocimiento científico, pero a veces con bastante intuición respecto a los enfermos mentales" (Jaramillo, 1970, p. 2).

Las condiciones de insalubridad del asilo eran complejas, pues este no contaba con alcantarillado, el agua era extraída de un pozo, el cual, según constataron análisis posteriores, estaba contaminado con parásitos. Se carecía de una adecuada asistencia médica y el principal tratamiento que se utilizaba eran los electrochoques. No había historias clínicas, sólo notas improvisadas en algún papel sobre el número de electrochoques aplicados a cada paciente y su posible enfermedad.

Como era usual en este tipo de instituciones, la demanda de pacientes superaba la oferta. En 1940, el Asilo San Isidro contaba con una capacidad de albergar noventa pacientes, pero las solicitudes de hospitalización superaban las cuatrocientas personas. De acuerdo con los reportes de Salud Pública durante los años cuarenta, hubo un incremento progresivo en la cantidad de enfermos mentales en el Departamento del Valle. La siguiente Tabla muestra la cantidad de personas que durante la década de los cuarenta consultaron el Asilo de dementes San Isidro:

Tabla 1. Enfermos reportados Asilo San Isidro 1940-1950

\begin{tabular}{cc}
\hline Año & Cantidad \\
\hline 1940 & 468 \\
1942 & 494 \\
1944 & 532 \\
1946 & 591 \\
1948 & 686 \\
1050 & 632 \\
\hline
\end{tabular}

Fuente: Orejuela (2014:60)

\footnotetext{
5 Antes de su fundación los enfermos mentales eran enviados al Hospital San Juan de Dios en Bogotá o al San Rafael en Pasto.

6 Una pavorosa y horripilante visión del manicomio de Cali. Relator. Marzo 25 de 1935. Pág. 2.

7 Contrato del Asilo de Meléndez con las Hermanas Hijas de la Caridad de San Vicente de Paul. 26 de agosto de 1946. Libro: 7. Archivo Documental del Hospital Psiquiátrico Universitario del Valle.
} 
En lo que respecta a la situación económica, el señor Fortunato Aljure, director del Departamento de Higiene, le describía al Dr. Alex Cobo director del Asilo en 1955, su punto de vista sobre la institución:

Usted que ha tenido la generosa preocupación de informarse e interesarse con inspecciones personales del estado actual del Asilo San Isidro, sabe a cabalidad la situación precaria de la institución y la penosa condición en que las limitadas condiciones físicas y económicas, obligan a mantener a sus 200 enfermos. El presupuesto con que cuenta la institución es de $\$ 5.800$ mensuales, incluyendo la nómina de las hermanas, choferes, enfermeros, etc., como solo parte de esta suma se invierte en alimentación, resulta que el presupuesto para la comida es muy inadecuado. Es evidente que con esta suma es imposible alimentar a los enfermos y el enfermo mental goza de buen apetito. Afortunadamente, contribuciones de alimentos por parte de particulares ayudan a calmar el hambre de los alienados. El presupuesto actual no contempla ni permite gastos de droga, haciendo necesario el uso de métodos crueles y anticuados para poder manejar enfermos agitados. Esta falta de elementos técnicos hace posible salvar de la rutina física y psíquica a hombres y mujeres que podrían ser mejorados ${ }^{8}$. ( Cobo, comunicación personal, 25 de octubre de 1955)

El testimonio del director de Higiene confirma el abandono en que se encontraban los enfermos mentales en el Asilo, tanto por la falta de recursos para ofrecerles un tratamiento adecuado, como por la inadecuada planta física con que contaba. Esta situación obligaba permanentemente a trasladar a los enfermos a Pasto, Bogotá o Medellín, lo que suponía altos costos de transporte que provenían del erario.

Sobre la situación del Asilo y la necesidad de contar con una infraestructura más adecuada, un Comunicado de la Beneficencia Pública en 1941, expresaba:

Para la Nación es de mucha conveniencia crear nuevos centros para la correcta atención de las enfermedades mentales, pues no todos los casos son de posible curación en climas altos, como está sucediendo al enviar a todos nuestros enfermos a Pasto o Bogotá y porque abre a la investigación científica, un nuevo medio para los profesionales de Cali, un campo cuya extensión beneficiaría a la raza colombiana. Para los familiares de los muchos enfermos mentales vallecaucanos será muchísimo más placentero saber que los tienen en sitios como Cali, cerca de sus hogares y con atenciones que ellos, como principales interesados, pueden comprobar, que en sitios distantes en donde la sola acción del gobierno no es suficiente para alcanzar los buenos resultados que con ella se persiguen. Económicamente al construir nuestro asilo, la Nación se libera de un pase que le significa el movilizar a los enfermos de la República a los sitios en donde ahora se concentran, aumentando, con los propios edificios que levantaremos, el patrimonio común de la República (Archivo Hospital Psiquiátrico Universitario del Valle, 1941).
Las críticas que empiezan a hacerse al asilo provienen de miembros de diferentes instituciones a nivel nacional y municipal, pero sobre todo de la prensa local que lleva a cabo una importante función de veeduría de las instituciones del Estado como cárceles, asilos y hospitales. La opinión que generan dichas críticas, ligadas, sobre todo, a los altos costos que tiene que pagar el Departamento por el traslado de enfermos a otras ciudades del país, la emergencia de nuevas situaciones como la creación de la Facultad de Salud de la Universidad del Valle en 1950 y el nombramiento de un médico, Alex Cobo ${ }^{9}$ como director del Asilo de dementes San Isidro, generarán lo que March y Olsen (1989, citado por Powell y Dimagio 1999) llaman oportunidad de cambio.

La oportunidad de cambio se da en momentos, en que los recursos acumulados por los actores, sus preferencias o necesidades de modificar sus costos de transacción, los conducen a apostar por una transformación mayor en las reglas de juego, en las estrategias de todos o buena parte de los actores relevantes. En el caso particular del Asilo, las condiciones arriba mencionadas permitieron la creación de una Junta, tal y como reza el Decreto No. 290 de 1950 de la Alcaldía de Cali ${ }^{10}$ , cuyo objetivo fue llevar a cabo la construcción del hospital psiquiátrico.

La emergencia de un nuevo campo organizacional: la Junta pro-construcción.

La nueva Junta proconstrucción creada para la edificación de un moderno hospital psiquiátrico en la ciudad, convocó un conjunto de organizaciones y de ciudadanos que se aliaron estructurando un campo organizacional encaminado a generar inicialmente, una nueva infraestructura para la atención de los enfermos mentales de la ciudad, pero también para mejorar el tratamiento y la asistencia clínica a dicha población. En este último aspecto fue clave la creación del Departamento de Psiquiatría en 1956. Las organizaciones que estructuraron el campo organizacional fueron: la Universidad del Valle, el Ministerio de Salud Pública, la Beneficencia del Valle del Cauca y el Concejo Municipal.

Sin embargo, más allá de instituciones, la Junta estuvo conformada por un conjunto de ciudadanos que conforman lo que Camacho y Guzmán (1990) denominan una hegemonía filantrópica, encargada de velar por el funcionamiento del Asilo y crear las condiciones económicas y políticas para la construcción del moderno Hospital Psiquiátrico. El reconocimiento y la legitimidad que adquieren estos grupos "forma parte del carácter de los sectores dominantes, se amolda a la ideología que impulsa y resalta sistemáticamente el civismo, el amor por la ciudad, los deberes de los dirigentes y el sentido de la pertenencia a la comunidad" (Camacho \& Guzmán, 1990, p.189).

\footnotetext{
8 Archivo Hospital Psiquiátrico Universitario del Valle, Correspondencias. Carta del Doctor Fortunato Aljure, director departamental de higiene del doctor Alex Cobo, octubre 25 de 1955. 9 Carta del Secretario de Gobierno Municipal a Alex Cobo. 27 de octubre de 1948. Libro: 9. Archivo Central del Hospital Psiquiátrico Universitario del Valle

10 Decreto No. 290 de 1950 del 11 de mayo (Copia). Libro: Gerencia. Decretos Varios. Estatuto orgánico. 1943 a 1949. Archivo Documental del Hospital Psiquiátrico Universitario del Valle.
} 
Tabla 2. Junta Directiva del Asilo San Isidro, 1954

\begin{tabular}{ll}
\hline \multicolumn{1}{c}{ Cargo } & \multicolumn{1}{c}{ Nombre } \\
\hline $\begin{array}{l}\text { Presidente } \\
\text { Vice-presidente }\end{array}$ & $\begin{array}{l}\text { Alex Cobo } \\
\text { Cesorero }\end{array}$ \\
Secretaría & $\begin{array}{l}\text { Germán Holguín } \\
\text { Clemencia Fajardo }\end{array}$ \\
Revisor fiscal & Rubén Orozco Micolta \\
& \\
Vocales & Ignacio Gutiérrez \\
& Jaime Lozano Henao \\
& Mauricio Hannaford \\
& Mercedes Lloreda de Garcés \\
& Clarita Restrepo de Delgado \\
\hline
\end{tabular}

Fuente: abril 15 de 1954. págs. 1 y 14. El País.

Entre los miembros de la Junta cabe destacar el papel realizado por algunos de sus miembros como: Clara Inés Suarez de Zawadsky, quien se desempeñaba como periodista cuando era miembro de la Junta, fue también directora de la Dirección Cultural del Departamento y estuvo asignada a los Consulados de Colombia en Holanda e Italia; así mismo, fue de gran importancia la labor desarrollada por Mercedes Lloreda de Garcés, la cual se desempeñó como abogada, miembro del Consejo Municipal por varias vigencias y de la Cámara de Representantes. Fue también miembro de la Acción Católica y participó activamente en la recuperación de los damnificados de la explosión del 7 de agosto de 1956 en Cali. Por su parte Jaime Lozano Henao, vocal de la Junta, fue un empresario y político que asumió la Alcaldía de Cali entre 1953-1955; fue también presidente de la Asociación de Cultivadores de Caña (ASOCAÑA) y finalmente, Alex Cobo, médico cirujano que se desempeñó como presidente de la Junta, entre otros.

Como lo plantea María del Pilar Peralta (2017): "Quienes componen la Junta movilizaban alianzas y recursos para obtener donaciones privadas y auxilios con entidades públicas para anticipar fondos y donaciones de camas, equipos tecnológicos e incluso, la gestión del espacio y servicios religiosos" ( $p$. $60)$. En otras palabras, ponen al servicio de la Junta su estatus, su profesión, pero sobre todo sus redes sociales, tal y como lo muestra el siguiente grafo realizado por Peralta:

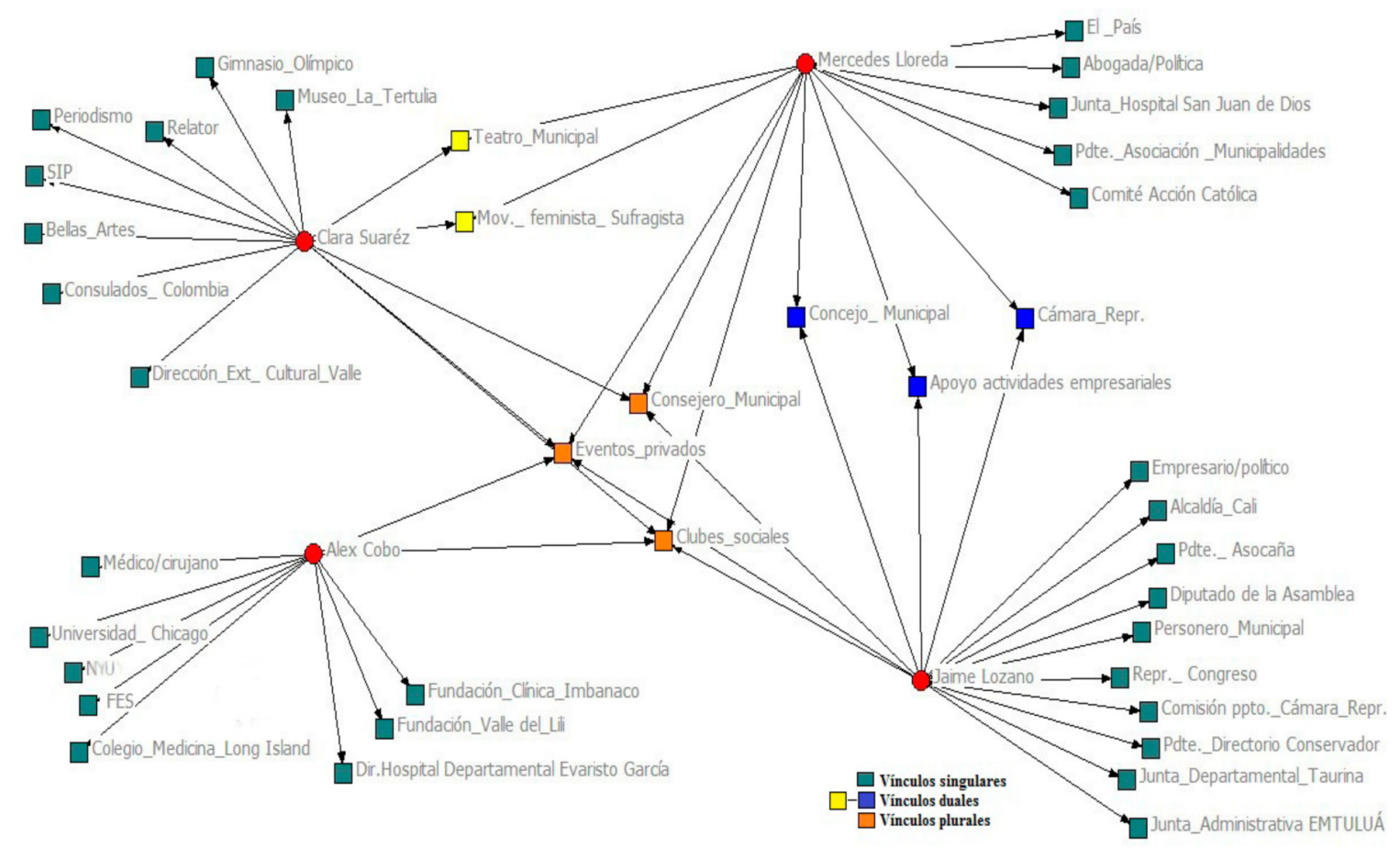

Fuente: María del Pilar Peralta (2017)

Figura 1. Vínculos y espacios de convergencia de miembros de la Junta

Los puntos en los que se encuentran los 4 miembros de la Junta son de carácter político (Consejo Municipal) y social (Clubes y eventos privados). Tal y como lo constata Collins (1981), las decisiones políticas se dan en el marco de procesos de sociabilidad y "vida en común" pero también en las "posiciones entrecruzadas en las juntas directivas, además de una constelación de relaciones económicas centradas alrededor de la familia" (Collins, 1981, p. 153).
Los espacios de sociabilidad en el que convergen los miembros influyentes de la sociedad caleña garantizan su cohesión e interrelación y su conformación como grupos. En dichos espacios como los clubes-según Edgar Vásquez-, las élites se reunían "para acordar negocios, socializar ideas sobre el manejo de la ciudad..." (Vásquez, 2012, p. 38).

La importancia de esta red de relaciones además de mostrar los puntos de articulación entre los miembros de la Junta, es 
que pone en evidencia "el aumento en el grado de interacción entre organizaciones en el campo" (Muñoz, 2014, p. 413 ); lo que permite pensar que los límites del campo organizativo, no se restringen, solamente, a los miembros de la Junta, sino que se extienden a sus zonas de influencia social, ámbito en el cual se encuentran empresas, otras instituciones del Estado y estructuras familiares.

La posibilidad de entrecruzar un amplio número de instituciones, familias e individuos potencializan las oportunidades de cambio, pues permiten la utilización de más recursos y la implementación de nuevas reglas, lo que facilita y agiliza el desarrollo de procesos de transformación a nivel social.

La Junta sustituyó paulatinamente a las hermanas en el manejo del Asilo y se dedicó inicialmente a conseguir dinero para mejorar la dotación y atención a los enfermos. Para ello realizaron actividades para recoger fondos (cenas, fiestas, etc.), pues los auxilios destinados por las instituciones públicas a la construcción del hospital no eran suficientes y rara vez se pagaban. Un ejemplo de este tipo de iniciativas es la carta dirigida por Mercedes Lloreda de Garcés y Elvira Hannaford (Miembros de la Junta Pro-Construcción) a los Miembros de la Junta Directiva del Club Colombia con motivo de la visita de la señorita Colombia a la ciudad:

La Junta Directiva del Asilo "San Isidro" [...] fue constituida para llevar a cabo la construcción de un hospital para enfermos mentales en esta ciudad. Como ustedes estarán informados no hay una Institución para el tratamiento de estos innumerables enfermos en todo el occidente de Colombia esta Junta con todas las atribuciones de Junta Directiva de tal establecimiento, con autoridad para solicitar y recaudar auxilios oficiales y particulares destinados a financiar su funcionamiento; acordar los presupuestos de gastos e inversión de los auxilios citados; reglamentar su régimen disciplinario interno y tomar las medidas que sean propias para la mejor dirección y funcionamiento del actual Asilo de Dementes11. (Lloreda, comunicación personal, 1957)

La obra proyectada por la Junta para el nuevo hospital tendría capacidad para 300 enfermos distribuidos de la siguiente forma:

130 hombres y 170 mujeres: 30 camas de enfermería para pacientes que sufrieran de enfermedades agudas, para enfermos quirúrgicos y para aquellos enfermos cuya sicosis los hubiera llevado a un extremo deterioro físico; 150 camas para sicóticos, graves, agitados y convalecientes; 110 camas para enfermos crónicos: 50 hombres y 60 mujeres. [...] Continúa además con: un equipo de rayos $X$, Electroencefalógrafo, aparato de electrochoques, sala de cirugía con dotación modesta pero adecuada para hacer cirugía general y cirugía del sistema nervioso, sala para convulsoterapia con insulina, facilidades modernas para hidroterapia, departamento de drogas. Una capilla y el apartamento del capellán (Orejuela, 2014, p. 68)

El diseño del hospital con salas para distintos tipos de terapia y tratamiento evidencia una transición en el sistema de creencias sobre la enfermedad mental, que no supone una ruptura radical con antiguas formas de tratar la enfermedad mental como las terapias convulsivas y la hidroterapia, sino más bien una mezcla de viejos y nuevos tratamientos, que muestra, sin embargo, una

11 Correspondencia Libro: 4. Archivo Cental del Hospital Psiquiátrico Universitario del Valle. tendencia en la que empezarán a prevalecer las drogas psiquiátricas, el TEC y la psicoterapia.

No obstante, el importante trabajo realizado en la consecución de fondos para la edificación del Hospital y el cuidado de los enfermos, una de las principales labores de la Junta fue incluir en la agenda publica el problema de la locura y la falta de instituciones adecuadas para el manejo y tratamiento de la enfermedad mental. Para ello se llevó a cabo un importante trabajo de visibilización del problema mediante una serie de noticias, columnas, crónicas y editoriales sobre el Hospital, que, si bien no inician, ni son tampoco escritas por todos los miembros de la Junta, algunos de ellos como Clara Suárez de Zawasdky, utilizan la prensa local para hacer referencia al problema y a la necesidad de generar condiciones para mejorar la atención de los pacientes en la ciudad. En otras palabras, va a permitir el "aumento de la carga de información" que se requiere para compatibilizar el discurso de la Junta con ciertas características del ambiente.

La importancia de esta estrategia va a rendir frutos porque introduce el problema de la salud mental en la agenda mediática y pública en la ciudad, a finales de los cuarenta y los cincuenta, lo que va a permitir que el proyecto del Hospital se vuelva una realidad. Con las contribuciones realizadas por el gobierno nacional y departamental y las donaciones particulares, se construyó en 1956 la primera etapa del hospital, "un edificio destinado a la administración, consulta externa, laboratorio, rayos $\mathrm{X}$, botica, servicio social, electroencefalografía, salas de cirugía, enfermería y salas de hospitalización para enfermos quirúrgicos" (Orejuela, 2014, p. 70).

Aunque a partir de 1956 se contó con un importante apoyo financiero por parte del Ministerio de Salud Pública, la Beneficencia del Valle, la Gobernación y la Alcaldía de Cali el hospital tuvo dificultades económicas para continuar con la construcción de la sede. Según el Presupuesto de Rentas del Asilo San Isidro para 1959, el Asilo contó con un monto de $\$ 72.600 .00$ en auxilios y $\$ 375.000 .00$ para la construcción del Hospital. En 1961, se lanzó una nueva campaña para recaudar fondos y terminar la edificación de pabellones para pacientes crónicos. Al igual que en otros momentos, se contó con el apoyo de la empresa privada de la región. Los aportes permitieron dotar 8 pabellones para enfermos, cuartos para médicos y empleados y camas para pacientes. En el siguiente cuadro se presentan los pabellones construidos distribuidos en salas, el carácter de cada una de ellas y el número de camas.

Tabla 3. Número de camas Hospital Psiquiátrico San Isidro

\begin{tabular}{ccc}
\hline Pabellón & Sexo & No. de camas \\
\hline Sala 1 & Pensionado mujeres & 20 \\
Sala 2 & Pensionado hombres & 17 \\
Sala 3 & Mujeres & 34 \\
Sala 4 & Hombres & 34 \\
Sala 5 & Mujeres & 34 \\
Sala 6 & Hombres & 34 \\
Sala 7 & Celda mujeres & 19 \\
Sala 8 & Celda hombres & 19 \\
Total Camas Hospital & 211 \\
\hline
\end{tabular}

Fuente: (Orejuela, 2014, pág.81) 
La producción y circulación de información sobre la enfermedad mental.

Un aspecto clave para crear las condiciones del cambio institucional y facilitar las actividades de la Junta como campo organizacional es, sin duda, la producción y circulación de información sobre el problema de la locura en general en la ciudad y en particular sobre las problemáticas que enfrenta el Asilo San Isidro para llevar a cabo su labor de cuidado a los enfermos mentales.
La producción de información que realizan medios de la época como el periódico El Relator, El Diario del Pacífico y El País contribuyen a generar un clima de opinión sobre el problema del Asilo San Isidro, pero logran, fundamentalmente, incluir en la agenda pública el problema de la "locura", lo que garantiza que se lleven a cabo acciones públicas y privadas para solucionar este problema en la ciudad.

Tabla 4. Publicaciones sobre el Asilo, la enfermedad mental y el Hospital en El Relator

\begin{tabular}{|c|c|c|}
\hline Título & Fecha & Tipo de documento \\
\hline Una pavorosa y horripilante visión del manicomio de Cali & Marzo 25 de 1935 & Crónica, pg 2 \\
\hline El problema de los locos & Enero 15 de 1940 & Noticia \\
\hline Se proyecta edificio de dementes & Mayo 12 de 1950 & Decreto pg 3 \\
\hline Cali necesita obras de acción social actual & Octubre 13 de 1951 & Columna pg 1 y 6 \\
\hline La preciosa Leonor en un acto de caridad & Enero 11 de 1952 & Noticia pg 6 \\
\hline Fue un intenso movimiento de locos en el Asilo de Medellín & Enero 21 de 1952 & Noticia pg 8 \\
\hline El loco por pena cuerdo & Febrero 6 de 1952 & Noticia pg 6 \\
\hline Alegría para el Asilo San Isidro & Febrero 12 de 1952 & Fotografía, pg 1 \\
\hline Por choque de dos locomotoras detenido un demente hace días & Septiembre 6 de 1952 & Noticia pg 8 \\
\hline Reunión ayer tarde en beneficio de los locos & Diciembre 4 de 1952 & Fotografía pg 4 \\
\hline Junta San Isidro-Gratísimo huésped & Diciembre 12 de 1952 & Noticia, pg 4 \\
\hline José María Rodríguez, condenado como enfermo mental & Marzo 5 de 1953 & Noticia pg 7 \\
\hline Congreso de psiquiatría y precursor en este campo & Marzo 5 de 1953 & Columna pg 7 \\
\hline Recibe garrotazos de un hermano loco en su casa & Marzo 25 de 1953 & Noticia, pg 8 \\
\hline Urge la construcción de pabellones en San Isidro & Mayo 5 de 1953 & Noticia, pg 10 \\
\hline Festival Hípico en el Campestre a beneficio del Asilo San Isidro & Julio 11 de 1953 & Invitación, pg 11 \\
\hline Problemas de matrimonio pueden producir demencia & Enero 16 de 1954 & Columna pg 2 \\
\hline Si usted quiere volverse loco, vaya al Asilo San Isidro & Febrero 23 de 1954 & Crónica, pg 7 \\
\hline Opinión sobre San Isidro -Ballet & Febrero 24 de 1954 & Columna, pg 4 \\
\hline Sanatorios para alienados & Abril 19 de 1954 & Noticia, pg 4 \\
\hline El Hospital Psiquiátrico & Mayo 8 de 1954 & Columna, pg 4 \\
\hline Cali va a construir grande Hospital Psiquiátrico & Mayo 8 de 1954 & Columna, pg 9 \\
\hline Un inmenso hospital para los enfermos mentales & Junio 2 de 1954 & Noticia, pg 3 \\
\hline Una versión Look para Relator (LSD) entrar al mundo de los locos & Noviembre 8 de 1954 & Reseña especial, pgs 5 y 9 \\
\hline Sobre los terrenos San Miguel y San Isidro & Noviembre 13 de 1954 & Noticia, pg 1 \\
\hline Junta Asilo San Isidro & Noviembre 22 de 1954 & Fotografía, pg 8 \\
\hline Desfiles a favor de San Isidro-Reflector Social & Febrero 21 de 1955 & Columna, pg 8 \\
\hline Aumentará fabricación de una gran droga para enfermedades mentales & Febrero 26 de 1955 & Noticia, pg 2 \\
\hline Manicomio para dementes peligrosos será construido & Mayo 9 de 1955 & Noticia, pg 3 \\
\hline Dos locos furiosos asaltaron una casa & Mayo 20 de 1955 & Noticia, pg 2 \\
\hline Sobre fondos San Isidro-Reflector Social & Junio 11 de 1955 & Columna, pg 8 \\
\hline San Isidro requiere ayuda & Junio 13 de 1955 & Anuncio, pg 10 \\
\hline Alarmante ola de locura entre los enfermos de Agua de Dios (Bogotá) & Junio 20 de 1955 & Noticia, pg 12 \\
\hline Sobre fondos San Isidro-Reflector Social & Junio 27 de 1955 & Columna, pg 8 \\
\hline Uso de drogas para la curación de enfermedades mentales se contempla & Diciembre 10 de 1955 & Noticia, pg 1 \\
\hline Navidad y Psiquiatría-Ballet & Diciembre 15 de 1955 & Columna, pg 4 \\
\hline A un manicomio irá por delito de lesiones, Hernando Carvajal & Diciembre 21 de 1955 & Noticia, pg 3 \\
\hline Exposición en la Tertulia sobre enfermedades mentales & Julio 11 de 1956 & Columna, pg 8 \\
\hline La salud mental y las enfermeras & Mayo 17 de 1957 & Columna, pg 12 \\
\hline
\end{tabular}

Fuente: El Relator 1935-1957 
Como es posible notar en la base de datos elaborada con diferentes tipos de piezas informativas del diario El Relator, hay entre 1951 y 1957, la publicación de una importante cantidad de noticias, columnas y crónicas que abordan de modo general el problema de la locura en la ciudad y en otras regiones del país. Los contenidos de estos discursos combinan desde notas judiciales, donde se enfatiza el carácter violento y agresivo de "los locos", pasando por informaciones generales sobre la evolución de la psiquiatría y los tratamientos modernos de la enfermedad mental, miradas compasivas y críticas sobre las instituciones que los albergan como el Asilo San Isidro, hasta actividades para la consecución de fondos adelantadas por los miembros de la Junta de esta misma institución.

Más allá de la diversidad de temas que difunde el periódico y los efectos que dichos contenidos tienen sobre las personas de la época, la publicación de piezas informativas sobre temas relacionados con la enfermedad mental es posible que haya contribuido a facilitar el trabajo de consecución de fondos y de mejoramiento de las condiciones de las instituciones mentales en la ciudad. En ese orden de ideas, la prensa cumplió no solo una función de catalizadora de la vida social, sino que reforzó nuevas percepciones sobre la realidad, que contribuyen a generar las condiciones para el cambio de actitudes y generar procesos de cambio social e institucional.

Cambios a nivel administrativo y en la práctica médica.

Paralelamente al trabajo desarrollado por la Junta en la construcción del nuevo hospital, empezaron a generarse cambios a nivel administrativo y en la praxis médica. A nivel administrativo, el paso del Asilo a Hospital implicó, en menos de 4 años, un incremento del personal a nivel administrativo y el dedicado a la atención de los pacientes, tal y como se evidencia en las dos siguientes tablas comparativas de dos periodos distintos 1956 y 1960

Tabla 5. Personal del Asilo San Isidro 1956

\begin{tabular}{ll}
\hline Cantidad & Cargo \\
\hline 1 & Síndico administrador \\
1 & Mecanógrafa secretaria \\
1 & Capellán \\
1 & Directora ecónoma \\
1 & Chofer \\
1 & Jefe de cocina \\
1 & Portero vigilante \\
1 & Peluquero vigilante \\
1 & Repostero \\
3 & Lavanderas \\
2 & Enfermeras jefas \\
3 & Enfermeras auxiliares \\
1 & Hortelano \\
3 & Costureras \\
5 & Cocineras \\
\hline
\end{tabular}

Fuente: Resolución No. 1 de noviembre de 1956
Tabla 6. Personal del Asilo San Isidro 1960

\begin{tabular}{|c|c|}
\hline Cantidad & Cargo \\
\hline \multicolumn{2}{|c|}{ Personal calificado, médicos y enfermeras } \\
\hline 1 & Director \\
\hline 1 & Sub director \\
\hline 4 & Médicos de medio tiempo \\
\hline 4 & Residentes \\
\hline 1 & Psicólogo \\
\hline 1 & Odontólogo \\
\hline 1 & Técnico de laboratorios \\
\hline 1 & Ginecólogo \\
\hline 1 & Radiólogo \\
\hline 1 & Internista \\
\hline 2 & Asistentes sociales \\
\hline 8 & Enfermeras graduadas \\
\hline 2 & Laborterapistas \\
\hline \multicolumn{2}{|c|}{ Personal administrativo } \\
\hline 1 & Administrador \\
\hline 1 & Contador \\
\hline 1 & Secretaria \\
\hline 1 & Jefe de Estadística \\
\hline 1 & Farmacéutico \\
\hline 2 & Motoristas \\
\hline 1 & Ecónoma \\
\hline 1 & Peluquero \\
\hline 1 & Empleado de información \\
\hline 4 & Vigilantes \\
\hline 1 & Empleado para reparaciones \\
\hline 1 & Cajero \\
\hline 1 & Portero \\
\hline 1 & Mensajero \\
\hline 1 & Capellán \\
\hline \multicolumn{2}{|c|}{ Personal auxiliar y de servicio doméstico } \\
\hline 20 & Auxiliares de enfermería \\
\hline 1 & Jefe de cocina \\
\hline 6 & Ayudantes de cocina \\
\hline 2 & Reposteros \\
\hline 6 & Aseadoras \\
\hline 4 & Lavadoras \\
\hline 4 & Costureras \\
\hline
\end{tabular}

Fuente: Plan de gastos Hospital Psiquiátrico San Isidro (1960)

Como lo muestran las Tablas 5 y 6, el paso del asilo a Hospital significó transformaciones profundas y muy rápidas, en la estructura administrativa. La primera tiene que ver con la cantidad de personal de ambas instituciones. Mientras el Asilo de dementes contaba para 1956 con 26 personas, dicha cantidad se triplicó en 1960, alcanzando 87 personas. Un segundo aspecto a reseñar, tiene que ver con la inserción de médicos, pues no se contaba en el asilo, salvo algunas enfermeras y el 
director, con profesionales de la salud y mucho menos con un especialista en la rama de la psiquiatría. En ese orden de ideas, un tercer aspecto a anotar es el cambio operado en la dirección de la institución, la cual empieza a recaer en personal científico y no en un Síndico ${ }^{12}$, como era usual en el esquema administrativo del Asilo. La presencia de personal en distintas ramas de la salud, como psicólogos, odontólogos, radiólogos, internistas, enfermeras y laborterapistas, muestra no sólo la diversidad de servicios que empieza a ofrecer el hospital, sino su carácter integrado, pues aborda diferentes dimensiones del problema de la salud, sin que el centro sea la enfermedad mental. Un cuarto y último aspecto en que vale la pena enfatizar, es la trasformación del hospital en una institución para la formación de médicos, pues cuenta con residentes que están cursando la especialidad en psiquiatría y desarrollando su práctica en este lugar.

Un aspecto que sin duda fue clave para llevar a cabo las transformaciones señaladas tiene que ver, además de las actividades desarrolladas por la Junta directiva del Asilo, con el papel desarrollado por el Departamento de Psiquiatría, que como se señala más adelante, va a encargarse de la dirección científica del hospital a partir de 1956. Este nuevo actor que va a insertarse en el nuevo campo organizacional conformado por la Junta, va a cambiar paulatinamente el modo de atención que hasta ese momento se le daba a los enfermos mentales en la ciudad, modificando así -al menos a nivel de la institución-, los sistemas de creencias sobre la locura, que como se mencionó supra, hace parte de un proceso más general en el que confluyen no tanto los psiquiatras, como la Organización Mundial de la Salud-OMS, que a través de sus políticas empiezan a cambiar la concepción sobre la enfermedad mental y el papel del personal médico y hospitalario en el tratamiento de este tipo de enfermedades.

Como se mencionó, con el propósito de crear servicios de atención en salud mental en los países miembros, la OMS inició una serie de eventos con expertos y consultores internacionales sobre la enfermedad mental en niños y personas adultas, en temas específicos como el alcoholismo, la epilepsia, así como en aspectos relacionados con la formación psiquiátrica de estudiantes de medicina y el rol que debían asumir sobre dicho campo, los funcionarios públicos. Paralelamente a la realización de eventos internacionales, la OMS destinó asesores y consultores especializados para que visitaran a las autoridades médicas de diversos países con el propósito de fomentar la implementación del programa de prevención e higiene mental. También dispuso becas para que médicos y miembros de los gobiernos asistieran a los eventos y conferencias programadas por la organización y realizaran estudios en psiquiatría infantil.

Los programas adelantados por la OMS en materia de formación a funcionarios y estudiantes de medicina con complementados con una política de creación de hospitales y centros de atención psiquiátricos públicos. "En el quinto informe publicado por el Comité de Expertos en la salud mental se estima que los servicios de salud mental deberían ser prestados por un centro relativamente pequeño, dotado para ofrecer servicios de consulta externa; dicha unidad podría funcionar de manera autónoma, ofrecer el servicio de hospital de día y en una de sus variantes, hacer parte de un hospital general"
(OMS, 1962). Este último modelo, fue el que se implementó en el Hospital Psiquiátrico Departamental del Valle.

En lo que respecta a la práctica médica, desde 1955 iniciaron las clases de psiquiatría en la Universidad del Valle y visitas de los estudiantes al Asilo. En el Acta de la Junta Directiva del 6 de enero de 1956, los médicos Carlos León y Ernesto Zambrano son nombrados como médicos visitadores del Asilo. En febrero del mismo año, el Departamento de Psiquiatría de la Facultad de Medicina de la Universidad del Valle fue designado, por esta misma entidad, como encargada de la dirección científica de la institución. En este mismo periodo, la Junta, con el apoyo del Departamento de Psiquiatría, pudo organizar un servicio de consulta externa con 8 consultorios, una oficina de historias clínicas, archivo y un departamento de servicio social.

Entre las primeras tareas realizadas por médicos y estudiantes referentes a la práctica médica y que contribuyeron a la organización del Hospital, fue la elaboración de historias clínicas. Para ello, utilizaron un modelo propuesto por el doctor Carlos A. León, que para ese entonces ya se desempeñaba como jefe del Departamento de Psiquiatría de la Universidad. En lo que tiene que ver con el enfoque teórico se integró al principio la perspectiva psicoanalítica y el enfoque psicodinámico de origen norteamericano. Al respecto señalan los doctores Jaramillo y Zambrano:

El enfoque teórico era el psicoanalítico, modificado de acuerdo con la psicodinámica adaptativa de Sander Rado, y yo recuerdo haber escrito páginas tras páginas de interpretaciones psicodinámicas sobre la historia de un esquizofrénico, al que naturalmente tratábamos con electrochoques" (Jaramillo, SF:2). (Jaramillo, Comunicación personal, $\sin$ fecha)

Otro avance significativo fue la liberación de los pacientes de sus celdas y el retiro de cadenas y grilletes que los sujetaban a manos y pies. Sin embargo, estos cambios, generaron resistencia por parte de las mismas hermanas, quienes habían sido hasta ese momento las encargadas de manejar el asilo y habían implementado "sus propias prácticas", inspiradas en preceptos morales y religiosos, que reñían con las nuevas propuestas planteadas por los médicos. Lo anterior estuvo acompañado de la dificultad para obtener el apoyo de los familiares de los pacientes, quienes en principio consideraron que una vez ingresados al asilo, estos no volverían a salir, despreocupándose por la recuperación y bienestar, descartando incluso, la posibilidad de recibirlos nuevamente en sus casas.

Tal y como lo reseña el periódico El País en 1956, los médicos Alex Cobo y Rómulo Mejía, ambos ocuparon cargos directos en la Junta y el Hospital respectivamente, asistían dos veces a la semana al Asilo y trataban a los enfermos con electrochoques. 


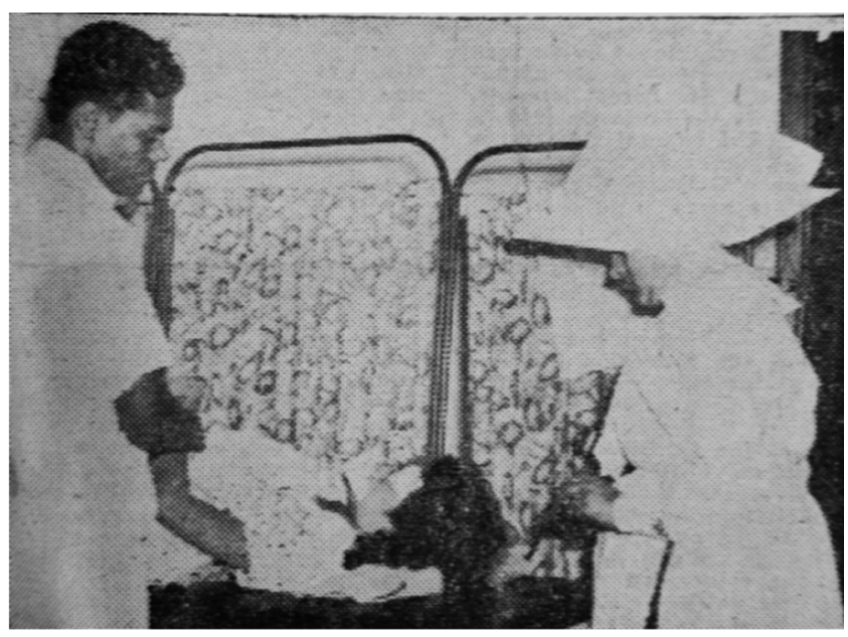

Fuente: Diario del Pacífico, enero 17 de 1952

Figura 2. Tratamiento de paciente con electrochoques

A nivel terapéutico, la terapia de electrochoques fue diversificada con el uso de la reserpina y la insulinoterapia. No obstante, estas dos últimas, fueron desplazadas paulatinamente por las fenotiazinas, que como se expuso supra, hicieron parte del gran descubrimiento farmacológico que transformó el tratamiento de la enfermedad mental.

En 1960, fue nombrado por la Junta Directiva del Hospital un médico adscrito al Departamento de Psiquiatría de la Universidad, el doctor Ernesto Zambrano. Su presencia le dio mayor control a la Universidad del Hospital y se tradujo en un mejoramiento del proceso de enseñanza y atención. Esto puede verse en la Tabla 5, que da cuenta del número de personas atendidas en el Hospital por hospitalización y consulta externa.

Tabla 7. Pacientes hospitalizados y por consulta externa en el $\mathrm{H}$. Psiquiátrico San Isidro

\begin{tabular}{ccc}
\hline Año & Hospitalización & Consulta externa \\
\hline 1956 & 310 & 194 \\
1957 & 369 & 148 \\
1958 & 337 & 259 \\
1959 & 557 & 529 \\
1960 & 801 & 1775 \\
1961 & 1109 & 2245 \\
1962 & 1057 & 3334 \\
1963 & 1117 & 5612 \\
1964 & 1492 & 7192 \\
1965 & 1377 & 8020 \\
1966 & 1859 & 8300 \\
1967 & 1798 & 8461 \\
1968 & 1898 & 8833 \\
\hline
\end{tabular}

Fuente: Jaramillo (SF)

La consulta externa y el hospital-día se convirtieron en una alternativa para tratar la enfermedad mental y sirvieron para descongestionar un hospital, que siguiendo la antigua inercia del modelo asilar, tuvo dificultades de sobrecupo al comien- zo, pues independientemente de la enfermedad, las personas eran internadas y se transformaban en pacientes permanentes de la institución. Esto fue posible gracias a la revolución farmacológica, que transformó el modo de tratar la enfermedad mental.

\section{Conclusiones}

El paso del Asilo al Hospital Psiquiátrico, supuso un cambio institucional que fue posible gracias al papel que desarrolló la Junta directiva del Asilo de dementes San Isidro durante las décadas 1940 y 1950. Esta junta, convocada por el doctor Alex Cobo, director del Asilo, permitió establecer alianzas con organizaciones privadas y públicas, que durante el periodo estudiado permitieron transformar la concepción que se tenía de la enfermedad mental, su atención y tratamiento en la ciudad.

Dicho cambio se produce, en un contexto de profundos cambios y discusiones sobre la salud mental en el mundo debido a las consecuencias generadas a nivel psicológico por la segunda guerra mundial. Este debate se traduce en la creación del Comité de Expertos de Salud Mental de la OMS en 1949, cuya finalidad fue la de crear un programa de salud mental para los países miembros. Las acciones de dicho comité se centraron en concitar la participación de expertos sobre el tema de la salud mental con el propósito de producir nuevas estrategias para abordar la enfermedad, sobre todo en el campo de la prevención, así como ofrecer el servicio de asesoría a los Estados para la implementación de Hospitales Psiquiátricos Públicos y formar el personal medico y de salud publica en las nuevas clasificaciones diagnósticas y terapéuticas.

En este sentido, la Junta conforma un grupo de actores que quiere cambiar las concepciones y la forma de tratamiento de la locura en la ciudad. Para ello pone en movimiento tres elementos básicos: la densidad de contactos inter-organizacionales, la organización financiada y el flujo de información y su consecuente "aprendizaje institucional" (Peters, 1999).

La densidad de contactos interorganizacionales es generada a partir de las redes personales e institucionales de los miembros de la Junta, los cuales ponen al servicio de la causa del Asilo de dementes, su estatuto profesional y social, lo que garantiza unir nuevos actores y ampliar el campo organizativo que soporta el proceso de transformación institucional.

En lo que respecta a la financiación, la Junta va a contar en sus inicios con muy pocos recursos, por eso los estatutos les confieren a sus miembros la posibilidad de consecución de fondos. Este trabajo de financiación lo van adelantar los miembros de la Junta a partir de la organización de actividades benéficas como espectáculos musicales y teatrales. $\mathrm{Pa}$ ralelamente a este proceso, la Alcaldía y otras instituciones empezaran a asignar partidas para el mantenimiento del Asilo y después para la construcción del hospital.

El flujo de información por su parte constituye un elemento esencial pues permite incluir el problema de la locura y el déficit institucional en la agenda pública, pero también crear las condiciones, mediante el suministro de una serie de diagnósticos y valoraciones sobre el problema, que allanaran el camino para llevar a cabo el cambio institucional.

Este proceso se implementó en muy poco tiempo y en un con- 
texto de profundas transformaciones a nivel internacional y nacional, entre las que vale la pena destacar: el desarrollo de una política sobre la salud mental desarrollada por la OMS, la publicación del manual diagnóstico de enfermedades mentales (DSM-I) en 1952, y el descubrimiento de la clorpromozapina, que revolucionó el tratamiento de la psicosis y la esquizofrenia. A nivel nacional se destaca la creación en 1946 del Ministerio de Salud Pública y un plan de desarrollo hospitalario para todo el país (Téllez, 2011, pp.106-108), así como las visitas de dos misiones médicas: la misión Humphreys o Médica Unitaria (1948) y la Lapham (1953), las cuales aconsejaron la creación de nuevas facultades de medicina y el desarrollo de especialidades médicas en el país. Estas crearon las condiciones para transformar la enseñanza de la medicina y el modo de tratar la enfermedad mental. Paradójicamente, todo ello se desarrolla en un momento de intensificación de la violencia en Colombia y del acceso al gobierno del General Gustavo Rojas Pinilla en 1954. Finalmente, a nivel local, fue muy importante la creación del Departamento de Psiquiatría en 1956, pues como ya se mencionó, sería dicho Departamento el encargado de la dirección científica del Hospital y su personal de profesores y estudiantes los conminados a ofrecer el servicio de atención psiquiátrica en el nuevo establecimiento. Todos estos aspectos crearon las condiciones para un cambio en el sistema de creencias sobre la salud mental, pero, sobre todo, fueron claves para la sustitución de un modelo asilar orientado excluir a "Ios locos" de la sociedad, mediante su confinamiento en una institución, por un modelo hospitalario centrado en la atención clínica y científica de los enfermos mentales.

La presencia de los médicos de la Facultad de Medicina de la Universidad del Valle y la creación del Departamento de Psiquiatría de este mismo centro educativo, jugaron un importante papel en las transformaciones que la Junta empezó a jalonar en la ciudad, pues ayudaron a que el nuevo Hospital se ajustara a los estándares internacionales y tuviera los espacios y la tecnología de un hospital mental moderno. Igualmente, gracias al nombramiento de los profesores del Departamento de Psiquiatría como encargados de la dirección científica del hospital, se logró incidir también la estructura administrativa y la praxis médica.

En ese orden de ideas, es posible afirmar que el cambio institucional llevado a cabo en el Hospital, fue resultado también del proceso de profesionalización de la psiquiatría en la región, pues supuso "la lucha colectiva de los miembros de una ocupación por definir las condiciones y métodos de su trabajo [...] y por establecer una base cognoscitiva y la legitimidad de su autonomía ocupacional" (DiMaggio y Powell,1999, p.113).

Los cambios operados en el Asilo y posterior Hospital Psiquiátrico San Isidro entre 1956 y 1970, constituyen una importante evidencia de las transformaciones realizadas en la praxis médica y en la base cognoscitiva de los psiquiatras durante el periodo estudiado. Particularmente, en lo que se refiere a la implementación de nuevas formas de clasificación de las enfermedades y del modo en que eran tratadas.

Estos nuevos tratamientos significaron una clara ruptura con el Asilo para dementes que se instauró en 1940 y en donde, prácticamente no existían historias clínicas y los métodos utilizados para tratar a los enfermos se basaban en su confinamiento en un estado deplorable de hacinamiento y maltrato (muchos enfermos permanecían amarrados y encadenados).
Todos estos cambios convirtieron al Hospital Psiquiátrico San Isidro en un importante centro de enseñanza para la formación de nuevos médicos y de atención para los enfermos mentales en el sur occidente colombiano.

\section{Referencias}

Alcaldía de Santiago de Cali. Decreto No. 290 de 1950

Asamblea Departamental del Valle del Cauca. Ordenanza No. 26 de 1940

Archivo Central del Hospital Psiquiátrico San Isidro

Archivo de Historias Clínicas Hospital Psiquiátrico San Isidro (1956-1970)

Beneficencia de Cundinamarca. Ordenanza No 2 de junio de 1888

Camacho, A. y Guzmán, A. (1990) La estructura social de la hegemonía filantrópica, el orden social y la violencia. Bogotá: Ediciones Foro Nacional por Colombia.

Collins, C. (1981) La prensa y el poder político en Colombia: tres ensayos. Colombia: Universidad del Valle.

APA (1952) Diagnostic and Statistical Manual I. Estados Unidos

APA (1968) Diagnostic and Statistical Manual II. Estados Unidos

Escalante, F. (1992) Ciudadanos imaginarios. México: El Colegio de México.

Farfán, G. (2007) El nuevo institucionalismo histórico y las políticas sociales POLIS 3 (I), 87-124

Hurtado, A. (1996) Régimen penitenciario y dispositivos carcelarios y de castigo bajo las reformas liberales en Cali. (Trabajo de pregrado) Universidad del Valle

Jaramillo, R (1970) Memorias sobre el desarrollo de la enseñanza de la Psiquiatría en Cali, dedicado a Carlos A. Léon En: Archivo del Departamento de Psiquiatría, Universidad del Valle.

López, L. (2009). Instituciones e Isomorfismo, implicaciones en la incertidumbre organizacional. Revista Mundo Económico y Empresarial, 7, 1-7. Recuperado de: http://revistas.ut.edu.co/index.php/rmee/issue/ view/79/showToc

March, J. y Olsen, J. (1984). The New Institutionalism: Organizational Factors in Political Life. The American Political Science Review. 78(3), 734-749.

March, J. y Olsen, J. (1997). El redescubrimiento de las instituciones: la base organizativa de la política. México: Colegio Nacional de Ciencias Políticas y Administración Pública A.C., Universidad Autónoma de Sinaloa y Fondo de Cultura Económica.

March, J. y Olsen, J. (2006). Elaborating the "New Institutionalism". En: R.A.W. Rhodes; S.A. Binder y B.A. Rockman (Ed.). The Oxford Handbook of Political Science (pp. 3-20). United States: Oxford University Press Inc.

Muñoz, R. (2014). Un enfoque integrador del cambio institucional en los hospitales públicos. En. Departamento de Producción y Desarrollo (E) Instituciones y Desarrollo (p. 405-429). México: UAM-X, CSH.

North, D. (1990) Institutions, Institutional Change and Economic Performance. Cambridge: Cambridge University Press

OMS (1962) L'OMS et la Santé Mentale 1949-1961, Recuperado de : https://apps.who.int/iris/bitstream/ handle/10665/258530/OMS-Sante-Mentale1949-1961-fre.pdf;jsessionid=4628C2B65D621F07 
69EE084F2A001210?sequence $=2$

Peralta, M. (2017) Elite cívica y filantrópica: La Junta pro-construcción Hospital Psiquiátrico Sa Isidro, Cali, 19501961, (Tesis de Maestría) Universidad del Valle

Peters, G. (1999) El nuevo institucionalismo: Teoría institucional en Ciencia política. España: Editorial Gedisa.

Plan de gastos Hospital Psiquiátrico San Isidro (1960)

Powell, W. y Dimaggio, P. (1999) El nuevo institucionalismo en el análisis Organizacional México: FCE.

Orejuela, D. (2014). La locura en Cali: De una mirada asistencial a una mirada clínica, el caso del Asilo San Isidro 1940-1970. (Tesis de pregrado) Universidad del VaIle, Cali, Colombia. Recuperado de: http://salud. univalle.edu.co/simposio/index.php/simposioX$\mathrm{VI} /$ Simposio/paper/view/360

Téllez, M. (2011) Reconstrucción histórica del proceso de creación del Ministerio de Salud Pública en Colombia (Tesis de Maestría) Universidad Nacional de Colombia, Bogotá. Recuperado de: http://www.bdigital.unal.edu.co/4138/

Torres, E. (2015) El nuevo institucionalismo: ¿hacia un nuevo paradigma? Estudios Políticos, No 34, México, 117137.

Vásquez, E. (2012) Cali en la primera mitad del siglo XX: mentalidades y sensibilidad. En: Historia de Cali: Siglo XX. Cultura, 3, Colombia: Universidad del Valle, Facultad de Humanidades.

Yepes, C. (2017). Aportes teórico-conceptuales acerca del cambio organizacional de la industria cafetera colombiana. Suma de Negocios 8, 19-30 